\title{
Mutual coupling in MIMO wireless systems: a rigorous network theory analysis
}

Michael A. Jensen

jensen@byu.edu

Jon W. Wallace

wall@ieee.org

Follow this and additional works at: https://scholarsarchive.byu.edu/facpub

Part of the Electrical and Computer Engineering Commons

\section{Original Publication Citation}

Jensen, M. A., and J. W. Wallace. "Mutual Coupling in MIMO Wireless Systems: A Rigorous Network Theory Analysis." Wireless Communications, IEEE Transactions on 3.4 (24): 1317-25

\section{BYU ScholarsArchive Citation}

Jensen, Michael A. and Wallace, Jon W., "Mutual coupling in MIMO wireless systems: a rigorous network theory analysis" (2004). Faculty Publications. 429.

https://scholarsarchive.byu.edu/facpub/429 


\title{
Mutual Coupling in MIMO Wireless Systems: A Rigorous Network Theory Analysis
}

\author{
Jon W. Wallace, Member, IEEE, and Michael A. Jensen, Senior Memebr, IEEE
}

\begin{abstract}
A new framework for the analysis of multiple-input multiple-output (MIMO) wireless systems is introduced to account for mutual coupling effects in the antenna arrays. The multiport interactions at transmit and receive are characterized by representing the channel using a scattering parameter matrix. A new power constraint that limits the average radiated power is also introduced. The capacity of the MIMO system with mutual coupling is defined as the maximum mutual information of the transmit and receive vectors over all possible transmit signaling and receive loading. Full-wave electromagnetic antenna simulations combined with a simple path-based channel model are used to demonstrate the utility of the method.
\end{abstract}

Index Terms-Antenna array mutual coupling, information theory, MIMO systems.

\section{INTRODUCTION}

$\mathbf{M}$ ULTIPLE-INPUT MULTIPLE-OUTPUT (MIMO) wireless systems, characterized by multiple antennas at the transmitter and receiver, have demonstrated the potential for increased capacity in rich multipath environments [1]-[3]. For example, when the channel matrix coefficients are well-represented as independent identically distributed (i.i.d.) complex Gaussian random variables, linear increase in capacity with the number of antennas is possible. This independence of the channel coefficients is generally achieved by constructing antenna arrays with wide inter-element spacings (several wavelengths). For many mobile subscriber units, such separations are often unrealistic.

Close antenna element spacing inevitably leads to mutual coupling [4], [5]. Generally speaking, this coupling means that current induced on one antenna produces a voltage at the terminals of nearby elements. Recent studies have demonstrated that two closely-spaced coupled dipoles exhibit a lower correlation coefficient than identically spaced uncoupled dipoles [6], [7] due to the modification of the coupled antenna radiation and reception patterns. Other studies have examined the effect of mutual coupling on pattern characteristics for a variety of communications applications [8]-[12].

While these prior studies have presented important findings concerning the effect of array mutual coupling on MIMO system performance, they have neglected two key concepts. First, coupling at the transmitter impacts computation of the

Manuscript received December 12, 2002; accepted March 11, 2003. The editor coordinating the review of this paper and approving it for publication is Dr. M. Shafi. This work was supported by the National Science Foundation (NSF) under Wireless Initiative Grant CCR 99-79452 and Information Technology Research Grant CCR-0081476.

The authors are with the Department of Electrical and Computer Engineering, Brigham Young University, Provo, UT 84602 USA.

Digital Object Identifier 10.1109/TWC.2004.830854 radiated power, a quantity that must be properly constrained in capacity formulations. Second, the power collection capability and pattern characteristics of coupled antennas depend on impedance matching of the array to the communication electronics, implying that the effective channel transfer matrix depends on loading. Therefore, a true definition of capacity requires the selection of optimal loading at the receiver.

This work overcomes these difficulties by applying an exact network theory framework to automatically account for mutual coupling in the analysis of point-to-point MIMO systems. This framework includes a new constraint that limits the actual radiated power for coupled transmit antennas. New expressions for capacity are derived that maximize the mutual information of transmit and receive signals over all possible loading networks, providing a true upper bound on system performance. The network theory framework also allows more flexibility in specifying the dominant source of noise in the system. Consequently, two realistic noise models are presented, and conclusions concerning their impact on capacity are provided. This new framework offers the appropriate tools for definitively answering questions about the impact of mutual coupling on MIMO capacity.

The organization of the paper is as follows. Section II presents the communication system network models and formulates the radiated power constraint for coupled transmit antennas. Section III discusses two realistic noise models and derives closed-form capacity results for the traditional and the new radiated power constraint. To demonstrate the application of the new capacity expressions, Section IV describes full-wave finite-difference time-domain (FDTD) simulations of coupled 2-element arrays and uses the results to compute capacity as a function of element spacing for a simple stochastic channel model. The results are also compared to existing methods for computing capacity in MIMO systems with mutual coupling.

\section{NETWORK ANALYSIS}

Array mutual coupling studies typically use impedance matrices to represent the antenna network [13], [14]. However, we have found the use of scattering parameter (S-parameter) matrices to be a more natural representation for the capacity formulation. This S-parameter description can be generally expressed as

$$
\bar{b}=\overline{\bar{S}} \bar{a}
$$

where the vectors $\bar{a}$ and $\bar{b}$ denote the complex envelopes of the inward-propagating and outward-propagating waves, respectively, and $\overline{\bar{S}}$ is the S-parameter matrix. We will adopt 


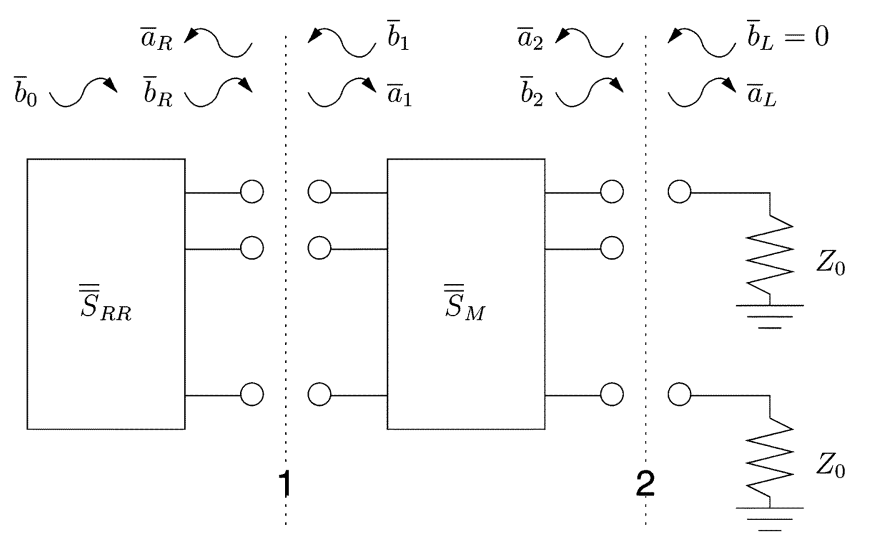

Fig. 1. Network model for the receive subsystem along with incident and reflected wave definitions.

the standard convention [15] that the total voltage and current on the $n$th port are given as $v_{n}=Z_{0}^{1 / 2}\left(a_{n}+b_{n}\right)$ and $i_{n}=Z_{0}^{-1 / 2}\left(a_{n}-b_{n}\right)$, where $Z_{0}$ is a chosen reference impedance used for computing the S-parameters. This normalization leads to the expression that the total power flowing into the $n$th port is simply $\left|a_{n}\right|^{2}-\left|b_{n}\right|^{2}$.

\section{A. Receive Subsystem: Matching Networks}

We first consider the network model depicted in Fig. 1 for the receive subsystem. This model treats the $N_{R}$-element antenna array as a source that creates the source wave vector $\bar{b}_{0}$ due to the received electromagnetic wave. This implies that if a load of characteristic impedance $Z_{0}$ is placed on each source port, the total power collected in the loads is equal to $\left\|\bar{b}_{0}\right\|^{2}=\bar{b}_{0}^{H} \bar{b}_{0}$, where $\{\cdot\}^{H}$ is the Hermitian or conjugate transpose operator. The source is further characterized by a (full) S-parameter matrix $\overline{\bar{S}}_{R R}$ such that $\bar{b}_{\underline{R}}=\bar{b}_{0}+\overline{\bar{S}}_{R R} \bar{a}_{R}$. A matching network with S-parameter matrix $\overline{\bar{S}}_{M}$ is used to maximize the power transfer from the source to the $N_{R}$ loads of resistance $Z_{0}$. We partition this matrix as

$$
\overline{\bar{S}}_{M}=\left[\begin{array}{ll}
\overline{\bar{S}}_{11} & \overline{\bar{S}}_{12} \\
\overline{\bar{S}}_{21} & \overline{\bar{S}}_{22}
\end{array}\right]
$$

where the subscripts 1 and 2 refer to input and output ports, respectively.

Ideally, the matching network is formed with passive, reactive elements so that it is lossless and reciprocal. If the network is lossless, ${ }_{\bar{S}_{M}}^{H} \overline{\bar{S}}_{M}=\overline{\bar{I}}$ where $\overline{\bar{I}}$ is the identity matrix. If it is reciprocal, $\left(\overline{\bar{S}}_{M}=\overline{\bar{S}}_{M}^{t}\right.$, where $\{\cdot\}^{t}$ is the transpose operator) we also have $\overline{\bar{S}}_{M} \overline{\bar{S}}_{M}^{H}=\overline{\bar{I}}$. It can be shown that the singular values of each $\overline{\bar{S}}_{i j}$ matrix lie on the range [0,1]. Also, if $\overline{\bar{S}}_{11}$ is set to be any matrix with singular values on the range $[0,1]$, one can use the matrix properties for lossless, reciprocal networks to specify the necessary form of $\overline{\bar{S}}_{12}, \overline{\bar{S}}_{21}$, and $\overline{\bar{S}}_{22}$ as shown in the Appendix.

Insertion of a lossless matching network between the source and the loads can increase the power collection if $\overline{\bar{S}}_{R R} \neq \overline{\overline{0}}$. In this case, the forward wave into the loads is

$$
\bar{b}_{2}=\overline{\bar{S}}_{21}\left(\overline{\bar{I}}-\overline{\bar{S}}_{R R} \overline{\bar{S}}_{11}\right)^{-1} \bar{b}_{0}
$$

and the total power collected is proportional to

$$
\begin{aligned}
P(\overline{\bar{S}}) & =\left\|\bar{b}_{2}\right\|^{2} \\
& =\bar{b}_{0}^{H}\left(\overline{\bar{I}}-\overline{\bar{S}}_{R R} \overline{\bar{S}}_{11}\right)^{(-1) H} \overline{\bar{S}}_{21}^{H} \overline{\bar{S}}_{21}\left(\overline{\bar{I}}-\overline{\bar{S}}_{R R} \overline{\bar{S}}_{11}\right)^{-1} \bar{b}_{0} .
\end{aligned}
$$

For a lossless network, we have the condition that $\overline{\bar{S}}_{11}^{H} \overline{\bar{S}}_{11}+$ $\overline{\bar{S}}_{21}^{H} \overline{\bar{S}}_{21}=\overline{\bar{I}}$, and the collected power becomes

$$
P(\overline{\bar{S}})=\bar{b}_{0}^{H} \overline{\bar{W}}\left(\overline{\bar{S}}_{11}\right) \bar{b}_{0}
$$

where

$$
\begin{aligned}
& \overline{\bar{W}}\left(\overline{\bar{S}}_{11}\right)=\left(\overline{\bar{I}}-\overline{\bar{S}}_{R R} \overline{\bar{S}}_{11}\right)^{(-1) H} \\
& \quad \times\left(\overline{\bar{I}}-\overline{\bar{S}}_{11} \overline{\bar{S}}_{11}\right)\left(\overline{\bar{I}}-\overline{\bar{S}}_{R R} \overline{\bar{S}}_{11}\right)^{-1} .
\end{aligned}
$$

The Appendix proves that for a fixed (but arbitrary) $\bar{b}_{0}$, the collected power in (5) is maximized when $\overline{\bar{S}}_{11}=\overline{\bar{S}}_{R R}^{H}$. In this case, (6) reduces to

$$
\overline{\bar{W}}\left(\overline{\bar{S}}_{11}\right)=\left(\overline{\bar{I}}-\overline{\bar{S}}_{R R} \overline{\bar{S}}_{R R}^{H}\right)^{(-1) H} .
$$

Setting the input $S$-parameters of the matching network equal to the conjugate transpose of the source S-parameters is the multivariate extension of conjugate matching [13], [16]. If $\overline{\bar{S}}_{M}$ is a passive (nonamplifying) network, the singular values of $\overline{\bar{S}}_{M}$ will all lie on the range $[0,1]$. In this case, one can show that a reciprocal, lossless network exists such that $\overline{\bar{S}}_{11}=\overline{\bar{S}}_{R R}^{H}$ is satisfied and the collected power is maximized.

\section{B. Transmit Subsystem: Constrained Radiated Power}

Traditional analyses of MIMO wireless systems have generally ignored the effect of mutual coupling on radiated power. Consider a transmit antenna array with $N_{T}$ elements and network S-parameters $\overline{\bar{S}}_{T T}$. The net power flowing into the network is $\left\|\bar{a}_{T}\right\|^{2}-\left\|\bar{b}_{T}\right\|^{2}$ which, for lossless antennas, equals the instantaneous radiated transmit power $P_{T}^{\text {inst }}$. Since $\bar{b}_{T}=$ $\overline{\bar{S}}_{T T} \bar{a}_{T}$, we have

$$
\begin{aligned}
P_{T}^{\text {inst }} & =\left\|\bar{a}_{T}\right\|^{2}-\left\|\overline{\bar{S}}_{T T} \bar{a}_{T}\right\|^{2} \\
& =\underbrace{\bar{a}_{T}^{H}}_{\bar{X}^{H}} \underbrace{\left(\overline{\bar{I}}-\overline{\bar{S}}_{T T}^{H} \overline{\bar{S}}_{T T}\right)}_{\overline{\bar{A}}} \underbrace{\bar{a}_{T}}_{\bar{X}}
\end{aligned}
$$

where $\overline{\bar{A}}$ is defined as the coherence matrix and $\bar{X}$ denotes a transmit signal vector. For zero mean signals, the average radiated power is given by

$$
P_{T}=\mathrm{E}\left\{P_{T}^{\text {inst }}\right\}=\operatorname{Tr}\left(\overline{\bar{K}}_{X} \overline{\bar{A}}\right)
$$

where $\overline{\bar{K}}_{X}=\mathrm{E}\left\{\bar{X} \bar{X}^{H}\right\}$ and $\mathrm{E}\{\cdot\}$ denotes an expectation. Section III-C considers the capacity under a transmitted power constraint based upon this formulation.

\section{Communication System Model}

Fig. 2 depicts an S-parameter block representation of a realistic communication system. The $N_{T}$ transmit antenna ports are excited by independent generators to create the inward at outward-traveling waves $\bar{a}_{T}$ and $\bar{b}_{T}$, respectively. The signals at the 


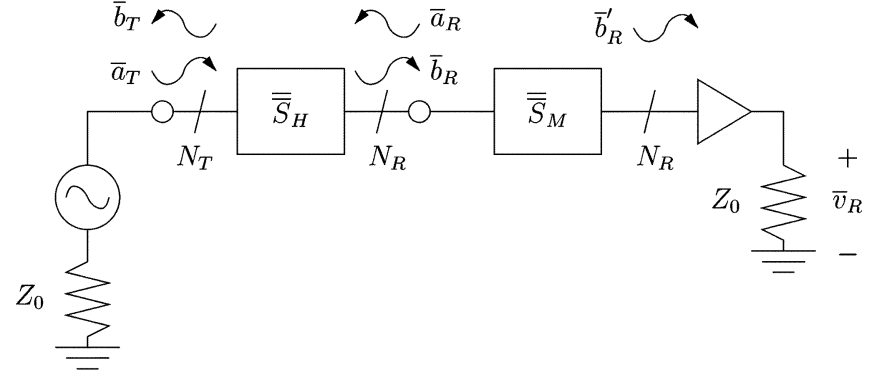

Fig. 2. Network model for the entire MIMO communication system.

$N_{R}$ receive antenna ports are similarly defined as $\bar{a}_{R}$ and $\bar{b}_{R}$. A unit gain element matched to the reference impedance $Z_{0}$ is included to allow the addition of noise in the receiver. Each port in the chain is terminated by a matched load $Z_{0}$, the voltage across which is $\bar{v}_{R}$. Because the output ports of the matching network $\left(\overline{\bar{S}}_{M}\right)$ are terminated in $Z_{0}$, only the outward-traveling wave $\bar{b}_{R}^{\prime}$ will exist at this point.

The antennas are embedded in a linear scattering medium so that the antenna/channel combination is represented by the block S-parameter matrix

$$
\left[\begin{array}{l}
\bar{b}_{T} \\
\bar{b}_{R}
\end{array}\right]=\underbrace{\left[\begin{array}{ll}
\overline{\bar{S}}_{T T} & \overline{\bar{S}}_{T R} \\
\overline{\bar{S}}_{R T} & \overline{\bar{S}}_{R R}
\end{array}\right]}_{\overline{\bar{S}}_{H}}\left[\begin{array}{l}
\bar{a}_{T} \\
\bar{a}_{R}
\end{array}\right] .
$$

For this analysis, we assume that $\overline{\bar{S}}_{T R}=\overline{\overline{0}}$, which means that power reflected from the receive antennas does not couple significantly back into the transmit antennas. This assumption indicates that the transmit and receive antennas are separated by a relatively large distance, presumably in the far-field of each other.

In the noiseless case, the sampled voltages are related to the transmit signal according to

$$
\underbrace{\bar{v}_{R}}_{\bar{Y}}=\underbrace{Z_{0}^{1 / 2} \overline{\bar{S}}_{21}\left(\overline{\bar{I}}-\overline{\bar{S}}_{R R} \overline{\bar{S}}_{11}\right)^{-1} \overline{\bar{S}}_{R T}}_{\overline{\bar{H}}\left(\overline{\bar{S}}_{M}\right)} \underbrace{\bar{a}_{T}}_{\bar{X}}
$$

where the dependence of the effective $N_{R} \times N_{T}$ channel matrix $\overline{\bar{H}}\left(\overline{\bar{S}}_{M}\right)$ on the matching network has been emphasized.

\section{MIMO CHANNEL CAPACITY}

The expression in (11) indicates that a true definition of capacity [17] requires maximization of the mutual information of $\bar{X}$ and $\bar{Y}$ not only over all possible transmit excitations $\left(\overline{\bar{K}}_{X}\right)$, but also over all allowed matching networks $\left(\overline{\bar{S}}_{M}\right)$. Since this maximization is dependent on the noise source, we consider two realistic noise models for microwave systems.

\section{A. Channel Noise Model}

If the dominant source of noise in the system is from the channel (co-channel interference, channel instability, cosmic radiation, etc.), we may neglect noise additions in the receiver. When no signal is present and the receive antenna ports are terminated in $Z_{0}$, we define the resulting forward traveling noise wave on the $i$ th receive port as $b_{R N, i}=Z_{0}^{-1 / 2} N_{i}$, where $N_{i}$ is an effective noise voltage. With the matching network inserted, the forward traveling wave becomes

$$
\bar{b}_{R N}=Z_{0}^{-1 / 2}\left(\overline{\bar{I}}-\overline{\bar{S}}_{R R} \overline{\bar{S}}_{11}\right)^{-1} \bar{N} .
$$

Superimposing the signal and noise vectors yields the result

$$
\bar{b}_{R}=\left(\overline{\bar{I}}-\overline{\bar{S}}_{R R} \overline{\bar{S}}_{11}\right)^{-1}\left(\overline{\bar{S}}_{R T} \bar{a}_{T}+Z_{0}^{-1 / 2} \bar{N}\right)
$$

leading to the channel equation

$$
\underbrace{\bar{v}_{R}}_{\bar{Y}}=\underbrace{\overline{\bar{S}}_{21}\left(\overline{\bar{I}}-\overline{\bar{S}}_{R R} \overline{\bar{S}}_{11}\right)^{-1}}_{\overline{\bar{P}}}(\underbrace{Z_{0}^{1 / 2} \overline{\bar{S}}_{R T}}_{\overline{\bar{H}}} \underbrace{\bar{a}_{T}}_{\bar{X}}+\bar{N}) .
$$

Assuming complex Gaussian signaling at the transmitter and Gaussian channel noise, the mutual information of $\bar{X}$ and $\bar{Y}$ is expressed as [17]

$$
\begin{aligned}
I(\bar{Y} ; \bar{X}) & =h(\bar{Y})-h(\bar{Y} \mid \bar{X}) \\
& =h(\bar{Y})-h(\overline{\bar{P}} \bar{N}) \\
& =\log _{2} \frac{\left|\overline{\bar{P}} \overline{\bar{S}}_{R T} \overline{\bar{K}}_{X} \overline{\bar{S}}_{R T}^{H} \overline{\bar{P}}^{H}+\overline{\bar{P}}_{\bar{K}_{N}} \overline{\bar{P}}^{H}\right|}{\left|\bar{P} \overline{\bar{K}}_{N} \overline{\bar{P}}^{H}\right|}
\end{aligned}
$$

where $h(\cdot)$ represents differential entropy and $\overline{\bar{K}}_{N}=$ $E\left\{\bar{N} \bar{N}^{H}\right\}$. If $\overline{\bar{P}}$ is nonsingular, this expression can be simplified to the form

$$
I(\bar{Y} ; \bar{X})=\log _{2} \frac{\left|\bar{S}_{R T} \overline{\bar{K}}_{X} \overline{\bar{S}}_{R T}^{H}+\overline{\bar{K}}_{N}\right|}{\left|\overline{\bar{K}}_{N}\right|} .
$$

This analysis indicates that, since the signal and noise undergo the same transformation in the matching network, matching does not change the mutual information. The only exception to this observation occurs when $\overline{\bar{P}}$ is singular, which implies loss of information in the network. For simplicity, the physical matching network can be removed $\left(\overline{\bar{S}}_{11}=\overline{\overline{0}}\right.$ and $\overline{\bar{S}}_{21}=\overline{\bar{I}}$ ) and, under the assumption of i.i.d. complex Gaussian noise with $\overline{\bar{K}}_{N}=\sigma^{2} \overline{\bar{I}}$, the mutual information becomes

$$
I(\bar{Y} ; \bar{X})=\log _{2}\left|\frac{\overline{\bar{S}}_{R T} \overline{\bar{K}}_{X} \overline{\bar{S}}_{R T}^{H}}{\sigma^{2}}+\overline{\bar{I}}\right| .
$$

Computation of the capacity for this equation is considered in Section III-C.

\section{B. Receiver Noise Model}

In single-user point-to-point transmission systems, the receiver front end is often the major source of noise. In this case, the amplifiers in Fig. 2 contribute the noise vector $\bar{N}$ at the output, leading to the relation

$$
\underbrace{\bar{v}_{R}}_{\bar{Y}}=\underbrace{Z_{0}^{1 / 2} \overline{\bar{S}}_{21}\left(\overline{\bar{I}}-\overline{\bar{S}}_{R R} \overline{\bar{S}}_{11}\right)^{-1} \overline{\bar{S}}_{R T}}_{\overline{\bar{H}\left(\overline{\bar{S}}_{M}\right)}} \underbrace{\bar{a}_{T}}_{\bar{X}}+\bar{N} .
$$


In this case, the mutual information expression is

$$
\begin{aligned}
& I(\bar{Y} ; \bar{X})=\log _{2}\left|\frac{\overline{\bar{H}}\left(\overline{\bar{S}}_{M}\right) \overline{\bar{K}}_{X} \overline{\bar{H}}\left(\overline{\bar{S}}_{M}\right)^{H}}{\sigma^{2}}+\overline{\bar{I}}\right| \\
& =\log _{2}\left|\frac{\overline{\bar{W}}\left(\overline{\bar{S}}_{11}\right) \overline{\bar{M}}}{\sigma^{2}}+\overline{\bar{I}}\right|
\end{aligned}
$$

where $\overline{\bar{W}}\left(\overline{\bar{S}}_{11}\right)$ is given in (6), the noise vector is i.i.d. complex Gaussian with $\overline{\bar{K}}_{N}=\sigma^{2} \overline{\bar{I}}$, and $\overline{\bar{M}}=\overline{\bar{S}}_{R T} \overline{\bar{K}}_{X} \overline{\bar{S}}_{R T}^{H}$. In general, $\overline{\bar{M}}$ is a Hermitian positive semi-definite matrix, so that we can use the eigenvalue decomposition (EVD) of $\overline{\bar{M}}$ to write

$$
\overline{\bar{M}}=\overline{\bar{\xi}}_{M} \overline{\bar{\Lambda}}_{M} \overline{\bar{\xi}}_{M}^{H}=\overline{\bar{M}}^{1 / 2} \overline{\bar{M}}^{(1 / 2) H}
$$

with $\overline{\bar{M}}^{1 / 2}=\overline{\bar{\xi}}_{M} \overline{\bar{\Lambda}}_{M}^{1 / 2}$. Thus, maximization of the mutual-information for a fixed (but arbitrary) $\overline{\bar{K}}_{X}$ requires maximization of

$$
I(\bar{Y} ; \bar{X})=\log _{2}\left|\frac{\overline{\bar{M}}^{(1 / 2) H} \overline{\bar{W}}\left(\overline{\bar{S}}_{11}\right) \overline{\bar{M}}^{1 / 2}}{\sigma^{2}}+\overline{\bar{I}}\right|
$$

over all possible values of $\overline{\bar{S}}_{11}$ and $\overline{\bar{K}}_{X}$.

This multivariate maximization is simplified by the fact that a simple conjugate match will always maximize (21) for fixed but arbitrary $\overline{\bar{K}}_{X}$. To show this, we use the result from Section II-A that

$$
\bar{x}^{H} \overline{\bar{W}}\left(\overline{\bar{S}}_{R R}^{H}\right) \bar{x} \geq \bar{x}^{H} \overline{\bar{W}}\left(\overline{\bar{S}}_{11}\right) \bar{x}
$$

for all possible values of $\overline{\bar{S}}_{11}$ and $\bar{x}$. Letting $\bar{x}=\overline{\bar{M}}^{1 / 2} \bar{y}$ and $\overline{\bar{W}}^{\prime}(\overline{\bar{S}})=\overline{\bar{M}}^{(1 / 2) H} \overline{\bar{W}}(\overline{\bar{S}}) \bar{M}^{1 / 2}$, we obtain

$$
\bar{y}^{H} \overline{\bar{W}}^{\prime}\left(\overline{\bar{S}}_{R R}^{H}\right) \bar{y} \geq \bar{y}^{H} \overline{\bar{W}}^{\prime}\left(\overline{\bar{S}}_{11}\right) \bar{y}
$$

and, therefore

$$
\bar{y}^{H}\left[\frac{\overline{\bar{W}}^{\prime}\left(\overline{\bar{S}}_{R R}^{H}\right)}{\sigma^{2}}+\overline{\bar{I}}\right] \bar{y} \geq \bar{y}^{H}\left[\frac{\overline{\bar{W}}^{\prime}\left(\overline{\bar{S}}_{11}\right)}{\sigma^{2}}+\overline{\bar{I}}\right] \bar{y} .
$$

For two positive definite matrices $\overline{\bar{A}}$ and $\overline{\bar{B}}$

$$
\bar{x}^{H} \overline{\bar{A}} \bar{x} \geq \bar{x}^{H} \overline{\bar{B}} \bar{x}, \quad \forall \bar{x}
$$

if and only if the singular values of $\overline{\bar{B}} \overline{\bar{A}}^{-1}$ are all less than or equal to 1 [18]. Hence, relation (24) implies that

$$
|\overline{\bar{A}}| \geq|\overline{\bar{B}}|
$$

leading to the conclusion [compare to (23)] that

$$
\left|\frac{\overline{\bar{W}}^{\prime}\left(\overline{\bar{S}}_{R R}^{H}\right)}{\sigma^{2}}+\overline{\bar{I}}\right| \geq\left|\frac{\overline{\bar{W}}^{\prime}\left(\overline{\bar{S}}_{11}\right)}{\sigma^{2}}+\overline{\bar{I}}\right|
$$

Therefore, for arbitrary $\overline{\bar{K}}_{X}, \overline{\bar{S}}_{11}=\overline{\bar{S}}_{R R}^{H}$ will maximize (21) and the mutual-information expression reduces to

$$
I(\bar{Y} ; \bar{X})=\log _{2}\left|\frac{Z_{0}}{\sigma^{2}}\left(\overline{\bar{I}}-\overline{\bar{S}}_{R R} \overline{\bar{S}}_{R R}^{H}\right)^{-1} \overline{\bar{S}}_{R T} \overline{\bar{K}}_{X} \overline{\bar{S}}_{R T}^{H}+\overline{\bar{I}}\right|
$$

Finding the value of $\overline{\bar{K}}_{X}$ that maximizes this equation will, therefore, lead to an expression for channel capacity.

Since $\left(\overline{\bar{I}}-\overline{\bar{S}}_{R R} \overline{\bar{S}}_{R R}{ }_{R}\right)$ is Hermitian and positive definite, (27) can be manipulated into the form

$$
I(\bar{Y} ; \bar{X})=\log _{2}\left|\frac{\overline{\bar{Q}} \overline{\bar{K}}_{X} \overline{\bar{Q}}^{H}}{\sigma^{2}}+\overline{\bar{I}}\right|
$$

where

$$
Z_{0}\left(\overline{\bar{I}}-\overline{\bar{S}}_{R R} \overline{\bar{S}}_{R R}^{H}\right)^{-1}=\overline{\bar{\xi}}_{D} \overline{\bar{\Lambda}}_{D} \overline{\bar{\xi}}_{D}^{H}
$$

is an EVD and

$$
\overline{\bar{Q}}=\overline{\bar{\Lambda}}_{D}^{1 / 2} \bar{\xi}_{D}^{H} \overline{\bar{S}}_{R T}
$$

The capacity for this case is considered in Section III-C.

\section{Modified Water-Filling ( $m w f$ ) Capacity}

The mutual information expressions (17) and (28) for the two different noise models are both of the general form

$$
I(\bar{Y} ; \bar{X})=\log _{2}\left|\frac{\overline{\bar{H}} \overline{\bar{K}}_{X} \overline{\bar{H}}^{H}}{\sigma^{2}}+\overline{\bar{I}}\right|
$$

where $\overline{\bar{H}}$ represents the channel matrix. For the traditional power constraint of $P_{T}=\operatorname{Tr}\left(\overline{\bar{K}}_{X}\right) \leq P_{\max }$, the capacity [17] of such a system can be formulated assuming that the transmitter has no knowledge (Uninformed Transmitter) [2] or perfect knowledge (Standard Water-Filling) [3] of the channel matrix $\overline{\bar{H}}$. When mutual coupling is properly considered, however, the power constraint based upon (9) becomes $P_{T}=\operatorname{Tr}\left(\overline{\bar{K}}_{X} \overline{\bar{A}}\right) \leq P_{\max }$. While the Uninformed Transmitter capacity expression remains the same under this change, the Water-Filling (wf) capacity solution must be modified in order to accommodate this new constraint.

Using the EVD $\overline{\bar{A}}=\overline{\bar{\xi}}_{A} \overline{\bar{\Lambda}}_{A} \overline{\bar{\xi}}_{A}$, we can rewrite the power constraint as $P_{T}=\operatorname{Tr}\left(\overline{\bar{K}}_{X}\right)$ with

$$
\overline{\bar{K}}_{X}^{A}=\overline{\bar{\Lambda}}_{A}^{1 / 2} \overline{\bar{\xi}}_{A}^{H} \overline{\bar{K}}_{X} \overline{\bar{\xi}}_{A} \overline{\bar{\Lambda}}_{A}^{1 / 2}
$$

Solving for $\overline{\bar{K}}_{X}$ and substituting into (31) leads to an expression that can be maximized using the standard wf approach. Unfortunately, this computation may be numerically sensitive when $\bar{A}$ has poor conditioning. 
To avoid these numerical instabilities, we instead first perform the singular value decomposition (SVD) $\overline{\bar{H}}=\overline{\bar{U}} \overline{\bar{S}} \overline{\bar{S}}{ }^{H}$ to obtain

$$
I(\bar{Y} ; \bar{X})=\log _{2}\left|\frac{\overline{\bar{S}} \overline{\bar{K}}_{X}^{\prime} \overline{\bar{S}}}{\sigma^{2}}+\overline{\bar{I}}\right|
$$

and express the resulting power constraint as $\operatorname{Tr}\left(\overline{\bar{K}}_{X}^{\prime} \overline{\bar{A}}^{\prime}\right)=P_{T}$, where $\overline{\bar{K}}_{X}^{\prime}=\overline{\bar{V}}^{H} \overline{\bar{K}}_{X} \overline{\bar{V}}$, and $\overline{\bar{A}}^{\prime}=\overline{\bar{V}}^{H} \overline{\bar{A}} \overline{\bar{V}}$. If $\bar{S}=\operatorname{diag}(\overline{\bar{S}})$, and the singular values are ordered from largest to smallest, we now consider only the $N_{S}$ largest singular values that satisfy $S_{1} / S_{i}<\rho$, where $\rho$ is some maximum allowed conditioning number. The matrices $\overline{\bar{K}}_{X}^{\prime}, \overline{\bar{S}}$, and $\overline{\bar{A}}^{\prime}$ are then truncated to contain only the leading $N_{S}$ rows and columns. In any model where $\overline{\bar{A}}$ and $\overline{\bar{H}}$ are consistent, this truncation ensures that $\overline{\bar{A}}$ has suitable conditioning, since transmit vectors yielding low radiated power will also yield low gain through the channel.

Next, we apply the substitution

$$
\overline{\bar{K}}_{X}^{\prime}=\overline{\bar{S}}^{-1} \overline{\bar{\xi}}_{Z} \overline{\bar{\Lambda}}_{Z}^{1 / 2} \overline{\bar{K}}_{X}^{Z} \overline{\bar{\Lambda}}_{Z}^{1 / 2} \overline{\bar{\xi}}_{Z} \overline{\bar{S}}^{-1}
$$

where we have performed the EVD of $\overline{\bar{S}} \overline{\bar{A}}^{\prime-1} \overline{\bar{S}}=\overline{\bar{\xi}}_{Z} \overline{\bar{\Lambda}}_{Z} \overline{\bar{\xi}}_{Z}$. The mutual information expression in (33) becomes

$$
I(\bar{Y} ; \bar{X})=\log _{2}\left|\frac{\bar{\Lambda}_{Z}^{1 / 2} \overline{\bar{K}}_{X}^{Z} \overline{\bar{\Lambda}}_{Z}^{1 / 2}}{\sigma^{2}}+\overline{\bar{I}}\right|
$$

with the power constraint $\operatorname{Tr}\left(\overline{\bar{K}}_{X}^{Z}\right)=P_{T}$. This expression may now be maximized using the wf solution approach [3].

\section{CAPACITY Simulations}

To demonstrate application of the analysis framework developed in this paper and to illustrate the impact of mutual coupling on the capacity of MIMO systems, we will explore transmit and receive arrays consisting of two coupled dipoles. Specifically, we focus on the receiver noise capacity expression from Section III-B. While analytical solutions exist for computing the impedance matrix for coupled dipoles, we are not aware of a good analytical model for predicting the effect of coupling on the radiation patterns, an integral part of the capacity analysis. As a result, full-wave FDTD simulations will be used to construct antenna network S-parameter descriptions and radiation patterns. These results are combined with a simple path-based channel model to construct the effective channel matrix.

\section{A. FDTD Antenna Simulations}

A likely candidate for characterizing coupled dipoles is the Moment Method since it is computationally efficient and wellsuited for wire antenna analysis [5]. However, the thin-wire assumption commonly used in this approach models the current as constant in azimuth around the wire radius, and as a result the simulations become less accurate for very close dipole spacings where this assumption is violated. As a result, a more detailed modeling of the antenna currents is required. In this case, we

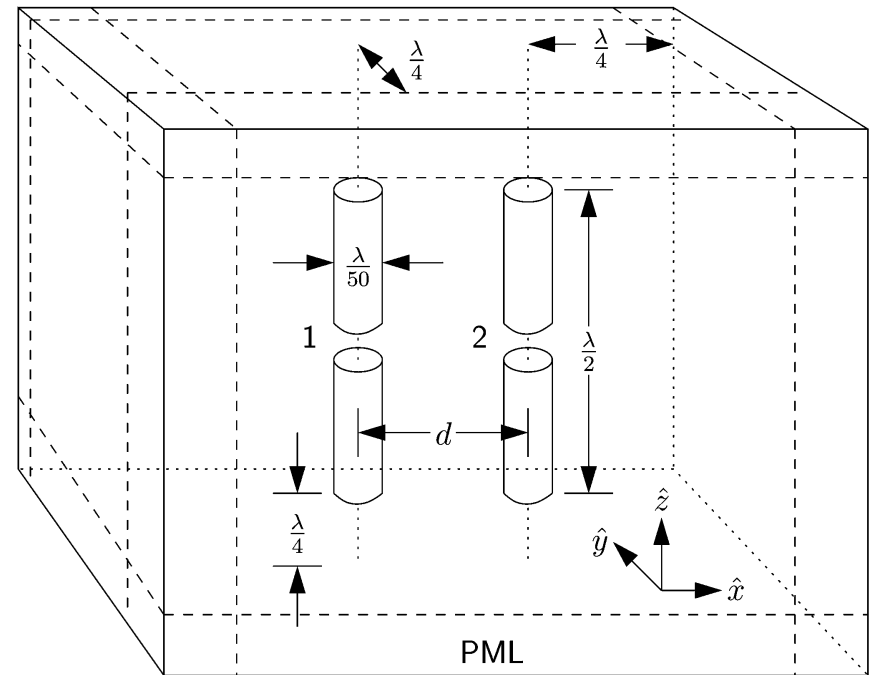

Fig. 3. FDTD simulation volume showing the geometry of the coupled dipole antennas.

have chosen to use FDTD [19], [20] for its computational simplicity, and have used very dense gridding in order to capture the detailed current behavior. This FDTD approach is also excellent for extending computations to more complex antennas, since it easily accommodates a wide variety of geometrical and material complexities. Obviously, other simulation approaches could be used for the computations. However, since the goal of this section is to demonstrate typical application of the developed framework rather than focus on the best computational technique suited for coupled antenna analysis, the choice of FDTD is suitable for purposes of simulating our model problem.

Fig. 3 depicts the FDTD geometry used for the coupled antenna simulations. Half-wave (total-length) dipoles with wire radius $0.01 \lambda$ and separated by a distance $d$ are located at the center of the domain. Because we are considering narrowband systems, single-frequency antenna excitation is used. The FDTD grid uses 80 cells per wavelength in the $\hat{z}$ direction and 200 cells per wavelength in the $\hat{x}$ and $\hat{y}$ directions to accurately model the current variations on the finite-radius wire for close antenna spacings. A quarter-wavelength buffer region is placed between the antennas and the terminating 8-cell perfectly matched layer absorbing boundary condition with a quadratic material profile [19]. The antennas are excited using a simple voltage source across the center FDTD cell of the wires, with resulting currents obtained using a contour integration around the source point [19], [20]. Each simulation runs for 10 sinusoidal cycles to allow the transient response to die down, after which current and field sampling is performed over one entire sinusoidal cycle. These quantities are then used to compute self and mutual impedances and radiation patterns [19], [20].

Fig. 4 plots the self and mutual impedance as a function of the element spacing. These results reveal a high degree of coupling, with the mutual impedance remaining significant even for antenna spacings greater than $1 \lambda$. Fig. 5 compares the radiated far-field patterns in the horizontal plane for four different dipole spacings. For this computation, the left antenna is driven by a source having an internal impedance of $Z_{11}^{*}$ and the right antenna is terminated with a load of impedance $Z_{11}^{*}$, where $Z_{11}$ is defined in Fig. 4. Each pattern is normalized to have unit mean. 


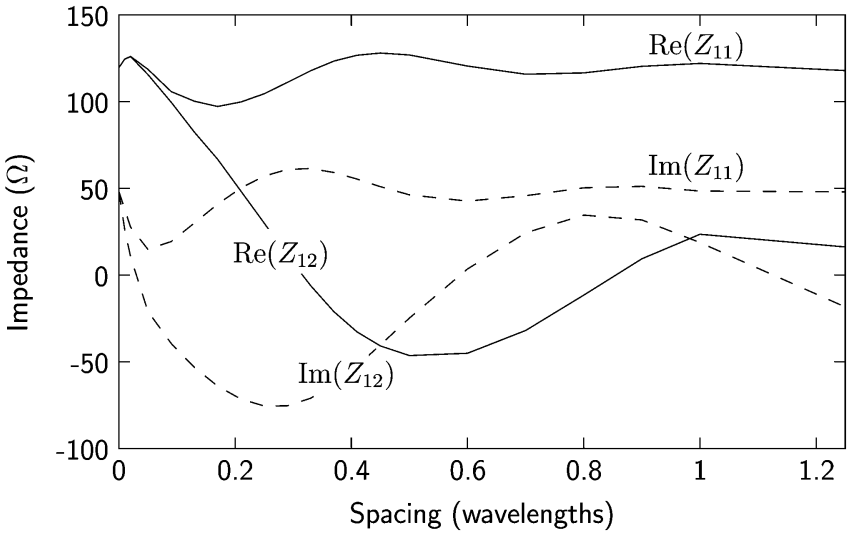

Fig. 4. Self and mutual dipole antenna impedances as a function of dipole separation $d$.

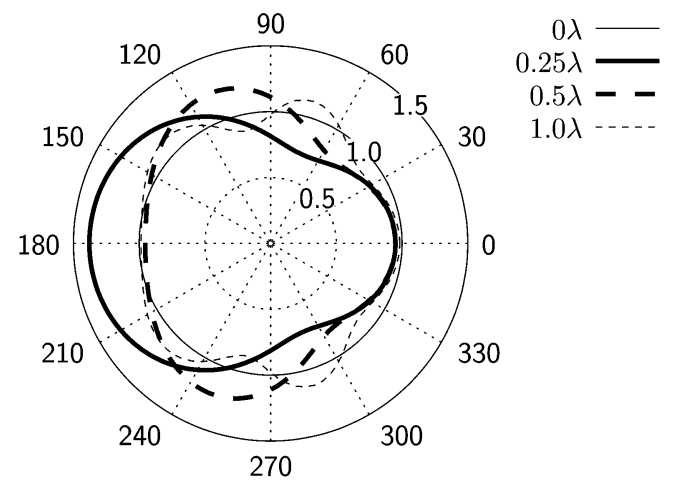

Fig. 5. Far-field coupled dipole patterns in the azimuthal plane for four different antenna spacings. The second antenna is terminated in $Z_{0}$.

These results indicate that the coupling noticeably impacts the patterns, particularly for smaller spacings.

\section{B. Path-Based Channel Model}

When the transmit and receive arrays and scattering objects are all in the far-field of one another, a simple path-based model can be used to approximate the channel. Here, we derive a simple two-dimensional single-polarization path-based model for use in the subsequent simulations.

Assuming a transmitter with $N_{T}$ antennas, we can write the total radiated far-field in the azimuthal plane as

$$
E^{T}\left(\theta^{T}\right)=\sum_{j=1}^{N_{T}} e_{j}^{T}\left(\theta^{T}\right) I_{j}^{T}
$$

where $\theta^{T}$ is the transmit azimuthal angle, $e_{j}^{T}\left(\theta^{T}\right)$ is the transmitted field per unit excitation current of the $j$ th antenna in the direction of $\theta^{T}$, and $I_{j}^{T}$ is the transmit excitation current applied at the $j$ th antenna terminals. We also assume a channel with $N_{A}$ paths for propagation from transmit to receive, with the $n$th path characterized by departure and arrival angles $\theta_{n}^{T}$ and $\theta_{n}^{R}$, respectively, and a complex channel gain $\beta_{n}$. When an $N_{R}$ element array is placed in the incident receive field, the voltage on the $i$ th receive element may be written as

$$
V_{i}^{R}=\sum_{j=1}^{N_{T}} \underbrace{\sum_{n=1}^{N_{A}} E_{i}^{R}\left(\theta_{n}^{R}\right) \beta_{n} e_{j}^{T}\left(\theta_{n}^{T}\right)}_{Z_{R T, i j}} I_{j}^{T}
$$

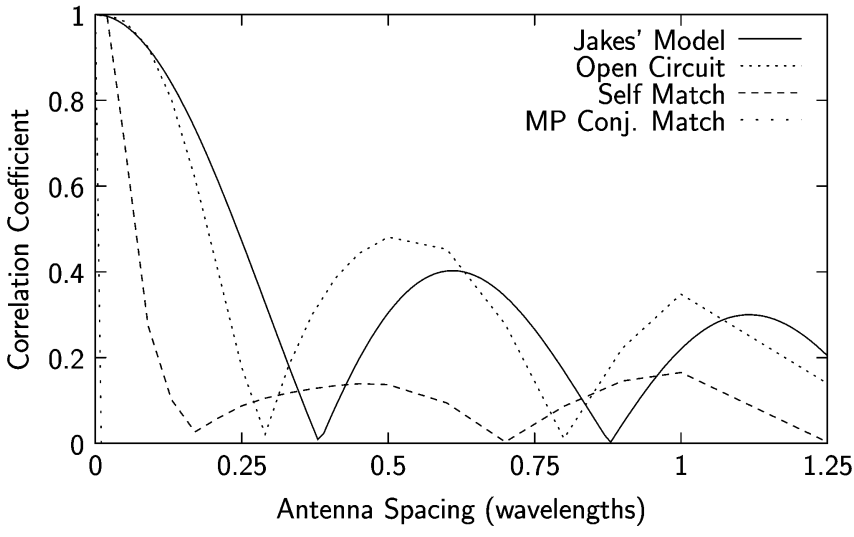

Fig. 6. Correlation coefficient versus dipole spacing for different antenna terminations compared to Jakes' model (neglecting mutual coupling).

where $E_{i}^{R}\left(\theta^{R}\right)$ is the reception pattern of the $i$ th receive antenna. The underbrace makes it clear that $\overline{\bar{Z}}_{R T}$ represents a trans-impedance matrix relating receive antenna voltages to transmit antenna currents. We also emphasize here that the radiation patterns are computed using the FDTD approach and, therefore, include the effects of the antenna mutual coupling.

The coupled transmit and receive arrays are characterized by the impedance matrices $\overline{\bar{Z}}_{T T}$ and $\overline{\bar{Z}}_{R R}$, respectively, which are also obtained from the FDTD simulations. Because we are neglecting feedback through the channel $\left(\overline{\bar{S}}_{T R}=\overline{\overline{0}}\right)$, we can individually convert $\overline{\bar{Z}}_{T T}$ and $\overline{\bar{Z}}_{R R}$ into $\overline{\bar{S}}_{T T}$ and $\overline{\bar{S}}_{R R}$ [15]. The block $\overline{\bar{S}}_{R T}$ is found by exciting an inward-traveling wave at the transmitter $\bar{a}_{T}$ and measuring the outward-traveling wave at the receiver $\bar{b}_{R}$. To ensure $\bar{a}_{R}=\overline{\overline{0}}$, the receive ports are terminated with loads $Z_{0}$. This procedure yields the relation

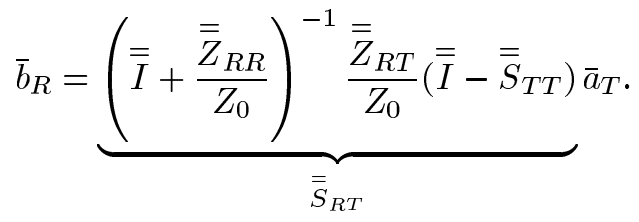

\section{Correlation and Power Collection}

The correlation coefficient of the complex baseband signals on two antennas is often used as a metric for assessing diversity performance. Correlation has also been used to draw conclusions about capacity, since a channel with transfer matrix coefficients that are complex Gaussian with low correlation exhibits high capacity. Here, we model the 2-element array with matching networks creating open-circuit, self-impedance matched load $\left(Z_{11}^{*}\right)$, and optimal multiport conjugate match terminations. The voltage correlation coefficient is computed as $E\left\{v_{1} v_{2}^{*}\right\}$, where $v_{i}, i \in\{1,2\}$ is the voltage at the $i$ th output port of the antenna matching network. The expectation is taken over plane wave azimuthal arrival angles which are uniformly distributed on $[0,2 \pi]$.

The resulting magnitudes of the correlation coefficients are plotted versus antenna spacing in Fig. 6. Surprisingly, the matching network offering a conjugate match always has an output correlation of zero for nonzero spacing. It is important to point out, however, that the optimal impedance match only specifies the block $\overline{\bar{S}}_{11}=\overline{\bar{S}}_{R R}$ of the matching network 


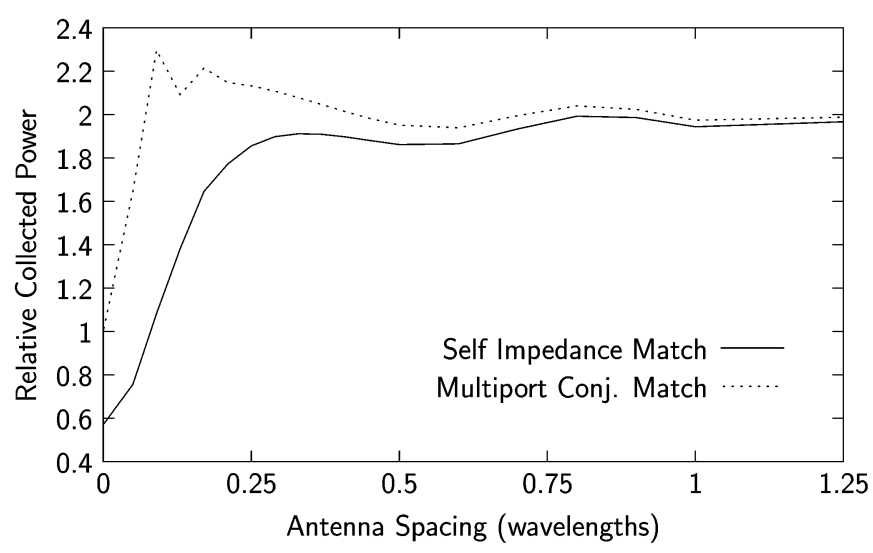

Fig. 7. Power collection (normalized to the power collected by a single dipole) versus antenna spacing for two dipoles assuming two different antenna terminations.

S-parameter matrix. Using the notation of the Appendix for a reciprocal network, we are still free to specify the unitary matrix $\overline{\bar{U}}_{S S}$, and here we have let $\overline{\bar{U}}_{S S}=\overline{\bar{I}}$ for simplicity. It can be shown analytically that this choice leads to perfect decorrelation for this case of symmetric dipoles. Physically, this occurs because the matching network modifies the radiation patterns for the individual elements such that they are orthogonal over $[0,2 \pi]$ in azimuth [13]. However, perfect decorrelation does not necessarily lead to improved diversity or MIMO performance since it also leads to unequal power in the independent matching network outputs. The other curves show that the correlation behavior is load dependent, with a self-impedance match exhibiting more decorrelation than the open circuit. The correlation curve assuming Jakes' propagation scenario (mutual coupling is neglected) is also shown for comparison [21].

Because capacity depends upon received signal power, the power collection capability of compact arrays is also of interest. Physical arguments suggest that as two antennas approach each other, the total collected power approaches that for a single antenna. To assess this, we examine the power collected by the coupled dipoles normalized by the power collected by a single dipole in the same environment for illumination by a single plane wave. In the computation, the collected power was computed for all azimuthal plane wave arrival angles for each spacing, and the resulting power levels were averaged. Fig. 7 plots this normalized collected power for optimal conjugate and suboptimal self-impedance match. Interestingly, only for very small spacings does the power collection drop significantly. The results also show that two closely-spaced antennas can actually collect more power than two widely separated (noninterfering) ones (i.e., the normalized collected power is greater than two). One reasonable explanation for this increased effective aperture of coupled dipoles is that a portion of the power scattered by each receiving antenna can be recaptured by the adjacent antenna, particularly when the matching network is appropriately implemented.

\section{Channel Capacity}

To demonstrate application of the analysis framework developed in this paper, we examine a system employing two transmit and two receive dipoles $(2 \times 2)$ and a channel consisting of

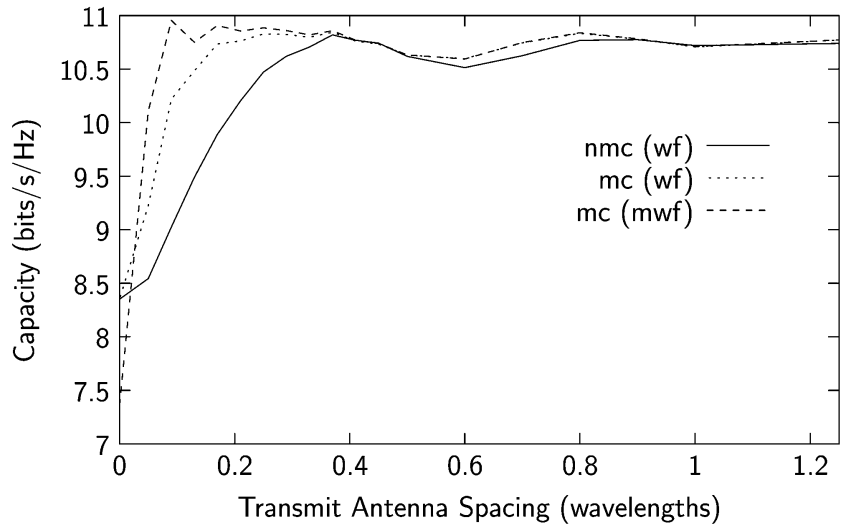

Fig. 8. Mean capacity versus transmit dipole antenna spacing for a $2 \times 2$ MIMO system with different assumptions on mutual coupling and transmit power constraints. The receive antennas are ideal and spaced at $d=1 \lambda$.

$N_{A}=4$ paths. The simple path-based channel model was combined with the full-wave FDTD antenna simulations (see Section IV-A) as discussed in Section IV-B to compute the channel matrix $\overline{\bar{S}}_{H}$. The ray arrival and departure angles were independent and uniformly distributed on $[0,2 \pi]$. The path gains $\beta_{n}$ were Rayleigh i.i.d. in amplitude and uniformly distributed in phase. For each channel realization, the noise power was fixed by first computing the average signal strength that would be received if one transmit and one receive antenna were present, with the averaging performed over random placement of the single transmit and receive antennas. The noise power was then computed to achieve a signal-to-noise ratio (SNR) of $20 \mathrm{~dB}$ relative to the average signal power. Mean capacity was computed over 7000 realizations for each antenna spacing.

To illustrate the effect of different transmit power constraints, simulations were run with ideal (no mutual coupling) receive antennas separated by $1 \lambda$. The transmit array was simulated with (mc) and without (nmc) mutual coupling, and the capacity was computed using standard (wf) and modified wf (mwf) solutions. The results of this study are shown in Fig. 8. For large spacings, the mutual coupling is low leading to identical capacity results. However, for very close spacings, the capacity computing using the mwf approach is actually higher than that obtained using the standard wf solution. Furthermore, the mwf solution properly accounts for the power loss observed as the antennas collapse to a single element, leading to the reduced capacity at $d=0$. Because the traditional power constraint does not incorporate the coherent interactions, it fails to properly predict this behavior.

We consider next the effect of mutual coupling at the receiver. In this case the receive antenna spacing was varied while the (ideal) transmit antennas were fixed at a separation of $1 \lambda$. A normalized channel analysis (norm) was performed by terminating the antennas with a self-impedance match and normalizing the channel transfer matrix to obtain exactly $20 \mathrm{~dB}$ average single-input single-output SNR [22] for each channel realization. This normalized channel analysis is similar to that considered in previous work [7]. Capacity was also computed with the new mutual coupling analysis with a constant noise giving an average of 20-dB SNR for the single antenna case with random placement as discussed at the beginning of this section.

Fig. 9 plots the average capacity versus spacing assuming mutual coupling with an optimal match (mc), no mutual coupling with an optimal match (nmc), and mutual coupling with 


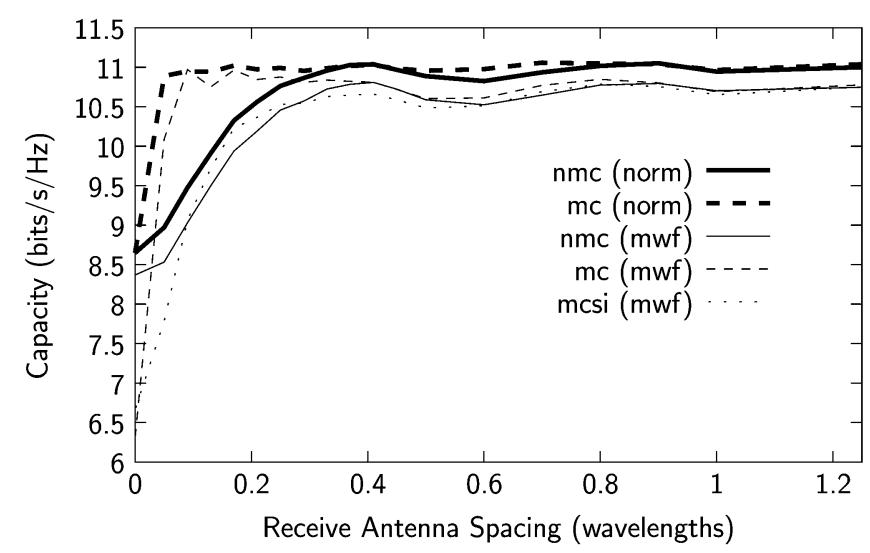

Fig. 9. Mean capacity versus receive antenna spacing for two different capacity computations and different antenna loads. The transmit antennas are ideal and spaced at $d=1 \lambda$

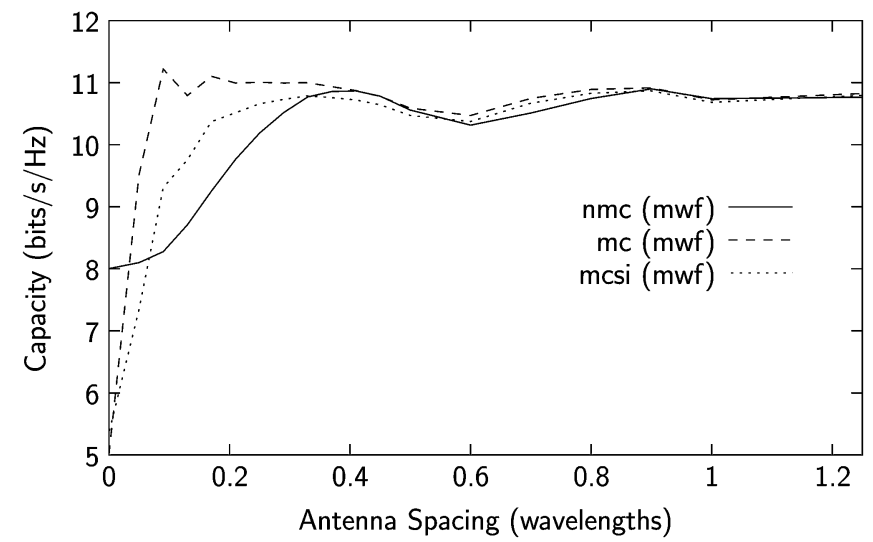

Fig. 10. Mean capacity versus transmit and receive antenna spacing for different coupling assumptions.

a suboptimal self-impedance match (mcsi). A small offset between the normalized and full analyses exists for large spacings since the SNR constraints are slightly different. In the normalized analysis, mutual coupling always provides a capacity benefit. In the full analysis, mutual coupling appears to provide nearly the same benefit as suggested by the normalized analysis. However, for very close spacings $(<0.1 \lambda)$, the capacity curve for the full analysis rolls off sooner than the normalized analysis. Finally, we note that although the simple self-impedance match performs well for large spacings, capacity degradation is apparent for spacings smaller than $0.25 \lambda$.

Fig. 10 demonstrates the combined effect of mutual coupling at transmit and receive. Here, the transmit and receive antenna spacings were equal and capacity was computed for ideal antennas (nmc), mutual coupling at transmit and receive with an optimal match (mc), and mcsi. For spacings between $0.1 \lambda$ and $0.3 \lambda$, mutual coupling provides an obvious capacity benefit. For spacings below $0.1 \lambda$, mutual coupling can actually degrade capacity. Finally, the suboptimal matching network yields a modest capacity degradation.

\section{CONCLUSION}

This paper has presented a rigorous network-theory framework for the analysis of mutual coupling in MIMO wireless communications. A detailed network model was used to develop a new mutual information expression and radiated power constraint accounting for this antenna coupling. Closed-form

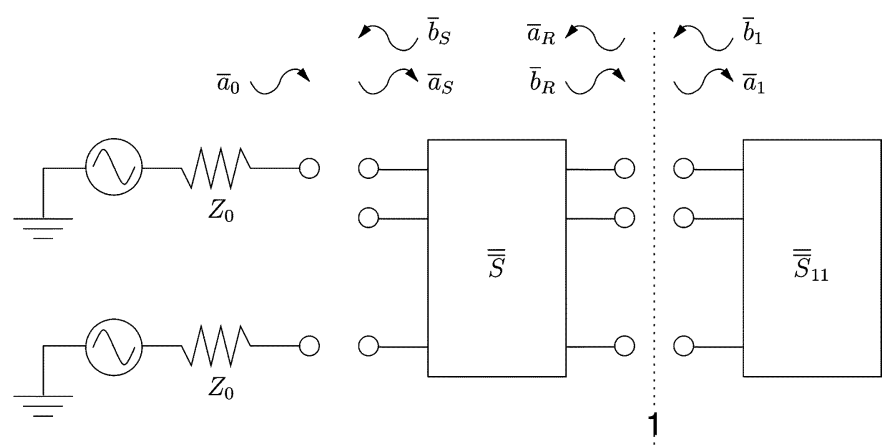

Fig. 11. Network model for the equivalent receive impedance matching problem.

derivation of the system capacity was made possible by relating the mutual information maximization problem to the multiport conjugate matching solution. Unlike previous analyses, this new method includes the effect of mutual coupling, and the resulting capacity expression provides a true upper bound on system performance.

The framework was used to analyze the impact of mutual coupling in a simple yet realistic $2 \times 2$ MIMO system by combining full-wave FDTD simulations with a path-based channel model. This simple example demonstrated the usefulness of the technique and provided some insight into the impact of coupling on MIMO performance. However, before more general conclusions can be drawn concerning the effect of mutual coupling, more extensive simulations using increased array sizes and various array configurations must be performed. Specifically, it is anticipated that mutual coupling will more significantly impact the performance of larger planar arrays. Fortunately, the tools developed here provide a comprehensive framework for systematically conducting these important studies.

\section{APPENDIX}

Consider the problem depicted in Fig. 1 . For any given $\bar{b}_{0}$, the power available from the source block $\overline{\bar{S}}_{R R}$ is fixed. We wish to choose the lossless matching block $\overline{\bar{S}}_{M}$ such that the power delivered to the load is maximized, regardless of the choice of $\bar{b}_{0}$.

We can cast this problem into the equivalent problem depicted in Fig. 11. Everything to the right of reference plane 1 has been replaced with the block $\overline{\bar{S}}_{11}$ (since $\bar{b}_{L}=\overline{\overline{0}}$ in Fig. 1). The source block is now represented with as many input ports as output ports, and the complete S-parameter matrix is given by

$$
\overline{\bar{S}}=\left[\begin{array}{ll}
\overline{\bar{S}}_{S S} & \overline{\bar{S}}_{S R} \\
\overline{\bar{S}}_{R S} & \overline{\bar{S}}_{R R}
\end{array}\right] .
$$

To make this problem equivalent to the initial one, we choose $\overline{\bar{S}}_{S S}, \overline{\bar{S}}_{S R}$, and $\overline{\bar{S}}_{R S}$ to make $\overline{\bar{S}}$ a lossless reciprocal network and set $\bar{a}_{0}=\bar{a}_{S}=\overline{\bar{S}}_{R S}^{-1} \bar{b}_{0}$. The relation for waves just to the left of plane 1 is

$$
\bar{b}_{R}=\overline{\bar{S}}_{R R} \bar{a}_{R}+\overline{\bar{S}}_{R S} \bar{a}_{S}=\overline{\bar{S}}_{R R} \bar{a}_{R}+\bar{b}_{0}
$$

which is precisely the same relation we had for the initial problem. Since $\overline{\bar{S}}$ is lossless, the power available to the load is $\left\|\bar{a}_{0}\right\|^{2}=\left\|\overline{\bar{S}}_{R S}^{-1} \bar{b}_{0}\right\|^{2}$. The load will collect all of this available 
power if we can choose $\overline{\bar{S}}_{11}$ such that the reflection $\bar{b}_{S}=\overline{\overline{0}}$. Relating the various inward- and outward-traveling waves, we have

$$
\bar{b}_{S}=\left[\overline{\bar{S}}_{S S} \overline{\bar{S}}_{R S}^{-1}+\overline{\bar{S}}_{S R}\left(\overline{\bar{I}}-\overline{\bar{S}}_{11} \overline{\bar{S}}_{R R}\right)^{-1} \overline{\bar{S}}_{11}\right] \bar{b}_{0} .
$$

In order to solve this problem, we require expressions that relate the subblocks of $\overline{\bar{S}}$ for a lossless reciprocal network. One may ensure that $\overline{\bar{S}}$ is lossless $(\overline{\bar{S}} \overline{\bar{S}}=\overline{\bar{I}})$ with the relations

$$
\begin{aligned}
& \overline{\bar{S}}_{R R}=\overline{\bar{U}}_{R R} \overline{\bar{\Lambda}}_{R R}^{1 / 2} \overline{\bar{V}}_{R R}^{H} \\
& \overline{\bar{S}}_{S R}=\overline{\bar{U}}_{S S} \overline{\bar{\Theta}}\left(\overline{\bar{I}}-\overline{\bar{\Lambda}}_{R R}\right)^{1 / 2} \overline{\bar{V}}_{R R} \\
& \overline{\bar{S}}_{R S}=-\overline{\bar{U}}_{R R} \overline{\bar{\Theta}}^{H}\left(\overline{\bar{I}}-\overline{\bar{\Lambda}}_{R R}\right)^{1 / 2} \overline{\bar{V}}_{S S} \\
& \overline{\bar{S}}_{S S}=\overline{\bar{U}}_{S S} \overline{\bar{\Lambda}}_{R R}^{1 / 2} \overline{\bar{V}}_{S S}^{H}
\end{aligned}
$$

where the first equation is the SVD of $\overline{\bar{S}}_{R R}, \overline{\bar{U}}_{S S}$ and $\overline{\bar{V}}_{S S}$ are arbitrary unitary matrices, and $\overline{\bar{\Theta}}$ is a complex diagonal matrix whose elements have unit magnitude with arbitrary phase. If we desire $\overline{\bar{S}}$ to be reciprocal $\left(\overline{\bar{S}}=\overline{\bar{S}}^{t}\right)$, we have the additional requirement that $\overline{\bar{U}}_{R R}=\overline{\bar{V}}_{R R}^{*}, \overline{\bar{U}}_{S S}=\overline{\bar{V}}_{S S}^{*},{ }_{=1 / 2=H}^{\text {and } \overline{\bar{\Theta}}}=j \overline{\bar{I}}$.

Using (42) and letting $\overline{\bar{S}}_{11}=\overline{\bar{S}}_{R R}=\overline{\bar{V}}_{R R} \bar{\Lambda}_{R R}^{1 / 2} \overline{\bar{U}}_{R R}^{H}$ in results in $\bar{b}_{S}=\overline{\bar{U}}_{S S} \overline{\bar{M}} \overline{\bar{U}}_{R R} \bar{b}_{0}$ with

$$
\begin{aligned}
\overline{\bar{M}}= & -\overline{\bar{\Lambda}}_{R R}^{1 / 2} \overline{\bar{V}}_{S S}^{H} \overline{\bar{V}}_{S S} \overline{\bar{\Theta}}\left(\overline{\bar{I}}-\overline{\bar{\Lambda}}_{R R}\right)^{-1 / 2} \\
& +\overline{\bar{\Theta}}\left(\overline{\bar{I}}-\overline{\bar{\Lambda}}_{R R}\right)^{1 / 2} \overline{\bar{V}}_{R R}^{H} \overline{\bar{V}}_{R R} \\
& \times\left(\overline{\bar{I}}-\overline{\bar{\Lambda}}_{R R}\right)^{-1} \overline{\bar{V}}_{R R}^{H} \overline{\bar{V}}_{R R} \overline{\bar{\Lambda}}_{R R}^{1 / 2}
\end{aligned}
$$

Upon canceling the unitary matrices, we find that $\overline{\bar{M}}$ is indeed the zero matrix, which ensures that $\bar{b}_{S}$ is the zero vector for any choice of $\bar{b}_{0}$. Therefore, the assignment $\overline{\bar{S}}_{11}=\overline{\bar{S}}_{R R}{ }_{R R}$ ensures that all available power is dissipated in the load, thus maximizing the collected receive power.

\section{REFERENCES}

[1] J. H. Winters, "On the capacity of radio communication systems with diversity in a Rayleigh fading environment," IEEE J. Select. Areas Commun., vol. SAC-5, pp. 871-878, June 1987.

[2] G. J. Foschini and M. J. Gans, "On limits of wireless communications in a fading environment when using multiple antennas," Wireless Personal Commun., vol. 6, pp. 311-335, Mar. 1998.

[3] G. G. Raleigh and J. M. Cioffi, "Spatio-temporal coding for wireless communication," IEEE Trans. Commun., vol. 46, pp. 357-366, Mar. 1998.

[4] J. L. Allen and B. L. Diamond, "Mutual Coupling in Array Antennas," Lincoln Laboratory, M.I.T., Lexington, MA, Tech. Rep. 424 (ESD-TR-66-443), 1966.

[5] C. A. Balanis, Antenna Theory: Analysis and Design: Wiley, 1997.

[6] J. Luo, J. R. Zeidler, and S. McLaughlin, "Performance analysis of compact antenna arrays with MRC in correlated Nakagami fading channels," IEEE Trans. Veh. Technol., vol. 50, pp. 267-277, Jan. 2001.

[7] T. Svantesson and A. Ranheim, "Mutual coupling effects on the capacity of multielement antenna systems," in Proc. IEEE ICASSP'2001, vol. 4, Salt Lake City, UT, May 7-11, 2001, pp. 2485-2488.

[8] W. C. Y. Lee, "Effect of mutual coupling on a mobile-radio maximum ratio diversity combiner with a large number of branches," IEEE Trans. Commun., vol. COMM-20, pp. 1188-1193, Dec. 1972.

[9] R. R. Ramirez and F. De Flaviis, "Mutual coupling study of linear polarized microstrip antennas for use in BLAST wireless communications architecture," in IEEE Antennats Propagation Soc. Int. Symp. Dig., vol. 2, Salt Lake City, UT, July 16-21, 2000, pp. 490-493.
[10] G. V. Tsoulos, "Experimental and theoretical capacity analysis of spacedivision multiple access (SDMA) with adaptive antennas," Proc. Inst. Elect. Eng Commun., vol. 146, no. 5, pp. 307-311, 1999.

[11] K. R. Dandekar, H. Ling, and G. Xu, "Effect of mutual coupling on direction finding in smart antenna applications," Electron. Lett., vol. 36 , pp. 1889-1891, Oct. 2000.

[12] A. M. Wyglinski and S. D. Blostein, "Mutual coupling and scattering effects on cellular CDMA systems using smart antennas," presented at the IEEE Vehicular Technology Conf. (IEEE Fall VTC2000), vol. 4, Boston, MA, Sept. 24-28, 2000, pp. 1656-1662.

[13] R. G. Vaughan and J. B. Andersen, "Antenna diversity in mobile communications," IEEE Trans. Veh. Technol., vol. VT-36, pp. 149-172, Nov. 1987.

[14] R. G. Vaughan and N. L. Scott, "Closely spaced monopoles for mobile communications," Radio Sci., vol. 28, pp. 1259-1266, Nov.-Dec. 1993.

[15] D. M. Pozar, Microwave Eng.: John Wiley \& Sons, 1998, ch. 4.

[16] H. A. Haus and R. B. Adler, Circuit Theory of Linear Noisy Networks. New York: Wiley, 1959.

[17] T. M. Cover and J. A. Thomas, Elements of Information Theory. New York: Wiley, 1991.

[18] R. Horn and C. Johnson, Matrix Analysis. Cambridge, U.K.: Cambridge Univ. Press, 1985, ch. 7

[19] S. C. Hagness and A. Taflove, Computational Electrodynamics: The Finite-Difference Time-Domain Method, Second Edition. Boston, MA Artech House, 2000.

[20] M. A. Jensen and Y. Rahmat-Samii, "Performance analysis of antennas for hand-held transceivers using FDTD," IEEE Trans. Antennas Propagat., vol. 42, pp. 1106-1113, Aug. 1994.

[21] W. C. Jakes, Microwave Mobile Communications. Piscataway, NJ: IEEE Press, 1993

[22] J. W. Wallace and M. A. Jensen, "Characteristics of measured $4 \times 4$ and $10 \times 10 \mathrm{MIMO}$ wireless channel data at 2.4-GHz," in Proc. IEEE Antennas Propagation Soc. Int. Symp., vol. 3, Boston, MA, July 8-13, 2001, pp. 96-99.

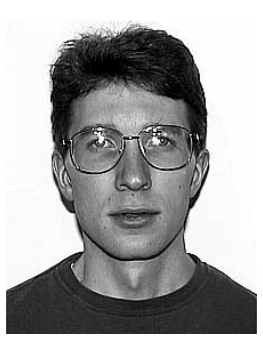

Jon W. Wallace (S'99-M'02) received the B.S. (summa cum laude) and $\mathrm{Ph} . \mathrm{D}$. degrees in electrical engineering from Brigham Young University (BYU), Provo, UT, in 1997 and 2002, respectively.

From 1995 to 1997, he worked as an associate of Novell, Inc., Provo, UT. During 1997, he was a Member of Technical Staff for Lucent Technologies, Denver, CO. From 1998 to 2002, he has worked as a graduate student researcher in the BYU Wireless Communications Laboratory. $\mathrm{He}$ is currently a research associate of the BYU Wireless Communications Laboratory and works in Vienna, Austria pursuing joint research with the Technical University of Vienna Mobile Communications Group. His research interests include wireless channel sounding and modeling, optical device modeling, and remote sensing.

Dr. Wallace received the National Science Foundation (NSF) Graduate Fellowship in 1998.

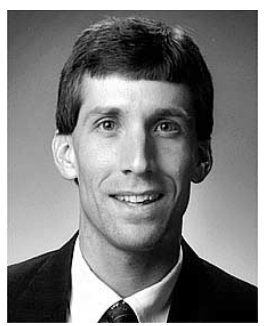

Michael A. Jensen (S'93-M'95-SM'01) received the B.S. (summa cum laude) and M.S. degrees in electrical engineering from Brigham Young University (BYU), Provo, UT, in 1990 and 1991, respectively, and the $\mathrm{Ph} . \mathrm{D}$. in electrical engineering at the University of California, Los Angeles, in 1994

From 1989 to 1991 he was a graduate Research Assistant in the Lasers and Optics Laboratory at BYU. From 1991 to 1994, he was a graduate student researcher in the Antenna Laboratory at UCLA. Since 1994, he has been at the Electrical and Computer Engineering Department at BYU where he is currently an Associate Professor. His main research interests include antennas and propagation for personal communications, microwave circuit design, radar remote sensing, numerical electromagnetics, and optical fiber communications. He is a member of Eta Kappa $\mathrm{Nu}$ and Tau Beta Pi.

Dr. Jensen received a National Science Foundation (NSF) Graduate Fellowship in 1990. He was awarded the best paper award at the 1994 IEEE International Symposium on Antennas and Propagation. He currently serves on the Joint Meetings Committee for the IEEE Antennas and Propagation Society, and served as the Technical Program Chair for the 2000 IEEE International Symposium on Antennas and Propagation. 